\title{
Androgen deprivation therapy affects BCL-2 expression in human prostate cancer
}

\author{
PAOLO FUZIO $^{1 *}$, PASQUALE DITONNO ${ }^{2 *}$, GIUSEPPE LUCARELLI ${ }^{2}$, \\ MICHELE BATTAGLIA ${ }^{2}$, CARLO BETTOCCHI ${ }^{2}$, TRABUCCO SENIA ${ }^{3}$ and ELDA PERLINO ${ }^{1}$ \\ ${ }^{1}$ Institute for Biomedical Technologies, ITB-CNR - Via G. Amendola, 122/D, 70126 Bari; ${ }^{2}$ Urology, Andrology \\ and Renal Transplantation Unit, Department of Emergency and Organ Transplantation, University of Bari; \\ ${ }^{3}$ Institute of Pathological Anatomy, University of Bari, Piazza Giulio Cesare 11, 70124 Bari, Italy
}

Received April 27, 2011; Accepted June 6, 2011

DOI: $10.3892 /$ ijo.2011.1140

\begin{abstract}
BCL-2 is an integral protein of the external mitochondrial membrane that inhibits cell apoptotic death. We investigated the effect of androgen deprivation therapy (ADT) on BCL-2 expression in prostate cancer tissues. We studied BCL-2 expression in vivo in prostate cancer tissues obtained from patients who underwent radical prostatectomy after neoadjuvant ADT, by Northern and Western blot analysis, and immunohistochemistry. Moreover, gene transcriptional activity was also measured by nuclear run-on experiments. We demonstrated an increase of BCL-2 mRNA expression in patients who underwent neoadjuvant ADT for 1 month in comparison to patients who had not received any therapy. Moreover, we demonstrated that there were no significant modifications of BCL-2 mRNA levels in patients who underwent neoadjuvant ADT for 3 and 6 months. Furthermore, BCL-2 protein levels in patients who underwent neoadjuvant ADT for 1 month were upregulated in comparison to patients who had not received any therapy. Immunohistochemical analysis showed a strong positivity of prostate cells depending on ADT administration for 1 month. Finally, transcriptional activity was not modified in patients who underwent neoadjuvant ADT, suggesting the absence of hormonal regulation on BCL-2 gene expression at the transcriptional level. Our data show that short-term administration of ADT interferes with BCL-2 expression, suggesting that androgen-mediated mechanisms may act through BCL-2-mediated apoptotic pathways. Moreover, since short-term ADT administration does not interfere with BCL-2 expression at the transcriptional level, the androgen-mediated mechanisms involving BCL-2 pathways, probably act at the posttranscriptional level.
\end{abstract}

Correspondence to: Dr Elda Perlino, Institute for Biomedical Technologies, ITB-CNR - Via G. Amendola, 122/D, 70126 Bari, Italy E-mail: elda.perlino@ba.itb.cnr.it

${ }^{*}$ Contributed equally

Key words: androgen deprivation therapy, BCL-2, expression regulation, prostate cancer

\section{Introduction}

Apoptosis is a morphologically distinct form of programmed cell death essential for normal development and tissue homeostasis (1). Aberrant regulation of this pathway is linked to multiple human diseases, including cancer, autoimmunity, neurodegenerative disorders and diabetes (2). The BCL-2 family of proteins constitutes a critical control point in apoptosis residing immediately upstream of irreversible cellular damage, where family members control the release of apoptogenic factors from mitochondria (2).

BCL-2 is an anti-apoptotic mediator that has been found to be involved in the molecular biology of a wide range of human cancers since its discovery as an oncoprotein implicated in human follicular lymphoma (3). It is known that the BCL-2 gene is a member of a multigene family, where the members function either to inhibit or to promote apoptosis (3). The protein encoded by this gene is a potent blocker of apoptosis and resides primarily in the parts of the endoplasmic reticulum, nuclear envelope and mitochondrial outer membrane conferring a survival advantage on cells expressing this oncoprotein (1). BCL-2 affects neoplastic cell proliferation by preventing apoptosis and therefore the normal cell turnover caused by physiological cell death mechanisms (3).

In normal prostate tissue, BCL-2 expression is restricted in the cytoplasm of basal epithelial cells, which are resistant to the effects of androgen deprivation $(4,5)$. On the other hand, the secretory epithelial cells, which lack detectable BCL-2 expression, undergo apoptosis following androgen deprivation $(4,5)$.

BCL-2 expression resulted higher in prostatic intraepithelial neoplasia and in the androgen-independent tumors emerging after androgen ablation treatment than in benign hyperplasia and untreated tumors (5).

Moreover, high levels of BCL-2 were demonstrated in LNCaP and PC-3 prostate carcinoma cell lines $(6,7)$. Increased levels of BCL-2 correlates with the development of prostate cancers, and suggested that this survival factor associate with a hormoneinsensitive, metastatic phenotype (8).

Prostate cancer cells are typically androgen-dependent and androgen ablation is the standard systemic therapy for this disease (9). Androgen deprivation induces programmed cell 
death in normal, hyperplastic, preneoplastic, and malignant prostatic epithelial cells (9). Androgen ablation provokes a selective pressure for clones that are genetically more likely to survive in an androgen depleted environment by activating alternative survival pathways. Pathways that lead to the up-regulation of BCL-2 have been proposed as one of the possible candidate mediating this process (9).

Gene transfection experiments using the androgen responsive prostate cancer cell line $\mathrm{LNCaP}$ that was modified to overexpress BCL-2, showed increased growth rate only when cultured in an androgen-ablated medium (10). The expression of BCL-2 protein protects $\mathrm{LNCaP}$ cells against apoptotic stimuli in vitro (10). Moreover, expression of BCL-2 enables $\mathrm{LNCaP}$ cells to form tumors in castrated male nude mice (10).

Immunohistochemical analysis of BCL-2 protein levels in prostate tumors have revealed a correlation between the presence of BCL-2 protein and resistance to anti-androgen therapy (4). Taken together, these observations suggest that elevated levels of BCL-2 protein may contribute to the progression of prostate cancers to a metastatic and hormone-insensitive state characterized by poor responses to chemotherapy.

The effects of dihydrotestosterone (DHT) on the regulation of BCL-2 expression in hormone sensitive LNCaP cell line, suggests that a consequence of androgen ablation therapy may be the de-repression of BCL-2 promoter and subsequent up regulation of BCL-2 mRNA and protein (11).

The involvement of BCL-2 in the progression of prostate cancer to androgen independence was further demonstrated by the use of antisense BCL-2 oligodeoxynucleotides (ODN). In fact inhibition of BCL-2 protein induces apoptosis and enhances chemosensitivity in human prostate cancer PC-3 and LNCaP cells $(12,13)$.

Taxane-induced (paclitaxel and its analogue docetaxel) phosphorylation of BCL-2 abolishes the potential antiapoptotic effect of BCL-2 (14). Considering that cancer cells that overexpress BCL-2 are sensitive to taxane-induced apoptosis, therapeutic response and survival benefit of hormone refractory prostate cancer (HRPC) patients treated with taxane-based chemotherapy may depend on the presence of BCL-2 protein. The up-regulated BCL-2 protein appears to be associated with the development of HRPC. However, the presence of BCL-2 protein, in turn, could indicate a survival benefit in HRPC patients scheduled for taxane-based chemotherapy (15). Moreover, another study confirmed that inhibition of BCL-2 function, by combined antisense BCL-2 ODN and chemotherapeutic agent after castration, delayed progression to androgen independent and inhibition of established androgen independent tumor growth in mice with Shionigi tumor $(16,17)$.

Therefore, the possibility of using gene therapy to downregulate BCL-2 as a treatment for prostate cancer is being considered and could be promising. Whether it is antisense, gene therapy or drug therapy, an effective anti-BCL-2 treatment of androgen-independent tumors may also potentiate the apoptosis inducing ability of other agents $(16,17)$ or radiotherapy (18), that otherwise cannot overcome the survival signal trigger by BCL-2 overexpression.

In this contest we decided to study the effect of neoadjuvant Androgen Deprivation Therapy (ADT) on the BCL-2 expression in patients affected by prostate cancer.

\section{Materials and methods}

Human prostate tissue collection and procurement. This study was performed using prostate specimens obtained from patients hospitalized at the Department of Emergency and Organ Transplantation-Urology, Andrology and Renal Transplantation Unit of the University of Bari (Italy). Informed consent was obtained from all patients.

Protocol-based tissue sampling techniques are used to procure tissue as soon as possible after removal from the patient ( $<20 \mathrm{~min}$ from time of removal at surgery to tissue freezing) while adequately preserving the specimen for pathological characterization (assessment of pathological stage, margin status, and grade).

Soon after removal, the prostate was placed on a cutting table and a single incision was made through the capsule. Then the capsule is stripped from the gland. This procedure permits procurement of tissue from the gland for banking while allowing for diagnostic evaluation of the entire prostate capsule tissue for surgical margin status and stage. A sample was taken and cut in two parts. This procedure generated two mirror image halves. One half was snap-frozen, and cryopreserved in liquid nitrogen for RNA extraction and immunoblotting analysis. The remaining tissue sample was fixed in $10 \%$ neutral-buffered formalin for 12 to $24 \mathrm{~h}$, embedded in paraffin, and stained with hematoxylin and eosin (H\&E) for histological evaluation. Identification of tumor areas in the frozen tissue was achieved by matching this tissue with $H \& E$ stained sections.

H\&E stained sections were reviewed, and the tumour grade, according to the Gleason criteria (19), was estimated for each tumour sample: Gleason score $<7 n=10$ and $\geq 7 n=29$.

RNA extraction and Northern blot analysis. Frozen tissue samples were pulverized and cellular RNA was extracted using the guanidinium isothiocyanate-cesium chloride procedure as previously described (20). Radiolabeled probes were generated using the Megaprime DNA labeling kit (Amersham), $100 \mu \mathrm{Ci}$ of $\alpha-{ }^{32} \mathrm{P}-\mathrm{dCTP}(3,000 \mathrm{Ci} / \mathrm{mmol}$, Amersham $)$ and $25 \mathrm{ng}$ of double-stranded $\approx 850$-bp fragment specific for the BCL-2. The specific $\approx 850$-bp BCL-2 fragment was generated by polymerase chain reaction using pcDNA3.1-BCL-2 plasmid as template and the resulting fragment was subcloned in the pcDNA3.1 vector. mRNA levels were normalized using ribosomal 28S rRNA, a constitutively expressed gene. For this purpose, blots were stripped in $0.1 \%$ boiling SDS and reprobed with the radiolabeled ${ }^{32} \mathrm{P}-28 \mathrm{~S}$ cDNA probe. Quantitative analysis was performed by densitometric scanning of the autoradiographs using a Bio-Rad GS-700 Imaging Densitometer (Bio-Rad, Richmond, CA); multiple exposures of the same Northern blots in a linear range were performed.

Nuclear run-on transcription assays. Isolation of nuclei and transcriptional rate assays were performed as previously described (21). Nylon N+ membranes (Amersham), containing $10 \mu \mathrm{g}$ of cDNA fragments previously bound by slot blot, were hybridized for $48 \mathrm{~h}$ at $42^{\circ} \mathrm{C}$ with $3.0 \times 10^{6} \mathrm{cpm} / \mathrm{ml}{ }^{32} \mathrm{P}$-labeled RNA obtained in the run-on transcription assay.

The following cDNA fragments were used in the nuclear run-on analysis: the $\approx 850$-bp specific BCL-2 fragment isolated by Eco-RI digestion from the pcDNA3.1-BCL-2 plasmid and the 
$1.3 \mathrm{~kb} 28 \mathrm{~S}$ fragment isolated by BamH1 digestion from the $\mathrm{p} 28 \mathrm{~S}$ plasmid (22).

After hybridization, the membranes were washed and then exposed to Kodak X-OMAT AR 5 film (Kodak, Rochester, NY, USA). Autoradiography of the RNA-DNA hybrids obtained after 7 days of exposure at $-80^{\circ} \mathrm{C}$ were analysed using a GS-700 Imaging Densitometer (Bio-Rad). All values were standardized according to the 28S rRNA signal used as the internal standard. The average of BCL-2 transcriptional activity in normal prostate biopsies derived from three patients was set at 100 (arbitrary units). BCL-2 transcriptional activity in neoplastic tissues was calculated as percentage of the transcriptional activity of the normal prostate.

Immunoblotting. Prostate frozen tissue samples obtained from radical cystoprostatectomy were homogenized and cellular proteins were extracted using the procedure as previously described (20). Briefly, $100 \mu \mathrm{g}$ of tissue extracts were electrophoresed on $10 \%$ SDS-polyacrylamide gel (PAGE) under reducing conditions and immunoblotting was carried out using: BCL-2 (Zymed Laboratories) antibody $(1 \mu \mathrm{g} / \mathrm{ml})$ and $\beta$-tubulin (Zymed Laboratories) antibody $(2 \mu \mathrm{g} / \mathrm{ml})$. In the first case the blots were incubated for $30 \mathrm{~min}$ at $4^{\circ} \mathrm{C}$ with a blocking solution composed of PBS pH 7.4 containing 5\% non-fat dry milk. Then the blots were incubated with the BCL-2 antibody for $16 \mathrm{~h}$ at $4^{\circ} \mathrm{C}$ in PBS containing 3\% non-fat dry milk. The membrane was then washed two times in water and incubated with horseradishperoxidase-conjugate goat affinity-purified antibody to mouse IgG (H+L) (Bio-Rad) 1/3.000 diluted with PBS containing 5\% non-fat dry milk for $90 \mathrm{~min}$ at room temperature. After two washes in water, one wash in PBS-T (PBS-Tween 0.05\%) and one wash in water again, the proteins were visualized using the Amersham enhanced chemiluminescent system according to the manufacturer's instructions.

Densitometric values for immunoreactive bands were quantified by using a GS-700 Imaging Densitometer (Bio-Rad). BCL-2 protein levels was calculated as percentage of the control (normal prostate) taken as 100 in arbitrary units, after normalization using $\beta$-tubulin as control for protein loading.

For each specimen, the mean value $( \pm$ SEM) of the results obtained in at least three experiments was calculated.

Histopathology and immunohistochemistry. The study material for histopathology included all surgical samples from which tissue fragments for the molecular investigations had been taken.

The surgical samples were fixed in $10 \%$ neutral buffered formalin for 12-24 h, embedded in paraffin, cut and stained with hematoxylin-eosin $(\mathrm{H} \& \mathrm{E})$. The histological preparations were reviewed by pathologist to compare the histological dating with the clinical dating in normal prostate, to specify the histological subtype of prostate carcinomas and to define tumor grade.

From selected cases (detailed in Table I) for which sufficient and representative amounts of tissues were available after morphological analysis, a single paraffin block per case was selected for immunostaining based on good morphological preservation. Three $\mu \mathrm{m}$ thick sections were cut, collected on positively-charged slides, dewaxed and rehydrated. Following quenching of endogenous peroxidase with $\mathrm{H}_{2} \mathrm{O}_{2}$ for $15 \mathrm{~min}$ at room temperature, the sections were immunostained for BCL-2 using a peroxidase-based detection system (En Vision,
Dako, Glostrup, Denmark) with an automated immunostainer (Autostainer, Dako). Prior to the staining procedure, the sections to be incubated with anti-BCL-2 antibody, were immersed in 1X EDTA buffer, $\mathrm{pH} 8.0$ and incubated at $98^{\circ} \mathrm{C}$ for $30 \mathrm{~min}$ in a water bath. A mouse monoclonal antibody against BCL-2 (Clone 100, Biogenex; dilution 1:500) was used as the primary antibody with overnight incubations at $4^{\circ} \mathrm{C}$. Sections of lymph node were used as positive control in our experiments.

Evaluation of BCL-2 immunoreactivity. In all cases the pathologist made the semi-quantitative evaluation by means the optical microscopy counting the relative number of immunoreactive cells in different microscopic fields (observed at x400 magnification). A semi-quantitative assessment on the overall number of cells immunoreactive was carried out. The extent of the immunoreactivity within each sections was expressed as the average percentage of immunoreactive cells in each sections and then it was semi-quantitatively scored.

Statistical analysis. All data are reported as the mean \pm SEM. Statistical analysis was performed using the ANOVA followed by Duncan post hoc test. All experiments were repeated at least three times.

\section{Results}

Effect of ADT on BCL-2 mRNA level. Steady-state level of BCL-2 mRNA was evaluated by Northern blot analysis starting from total RNA isolated from 34 prostate tissues (Table I).

We identified three groups of patients: the first group included 4 patients (N1-N4; median age at surgery: $74 \pm 6$ years) with a normal prostate who underwent radical cysto-prostatectomy for bladder carcinoma not involving the prostate. The second group included 21 patients (K1-K21; median age at surgery: $69 \pm 7$ years) with prostate cancer, who underwent radical prostatectomy.

The third group included 18 patients (TK1-TK18; median age at surgery: $68 \pm 5$ years) affected by prostate cancer who had undergone neoadjuvant ADT for 1 month (11 patients: TK1-TK11); 3 months (3 patients: TK12-TK14) and 6 months (4 patients: TK15-TK18) respectively.

The ADT consisted for all the patients of the association of a Luteinizing Hormone Releasing Hormone analog (goserelin depot, $3.6 \mathrm{mg} / \mathrm{q} 28$ days) and a non-steroidal anti-androgen (bicalutamide, $50 \mathrm{mg} /$ day). The median preoperative PSA level was $13.6 \mathrm{ng} / \mathrm{ml}$ (range: 6.1 to $18.6 \mathrm{ng} / \mathrm{ml}$ ) and $9.8 \mathrm{ng} / \mathrm{ml}$ (range: 2.2 to $16.3 \mathrm{ng} / \mathrm{ml}$ ) for patients in groups $\mathrm{K}$ and $\mathrm{TK}$, respectively.

Fig. 1A shows the result of a typical Northern hybridization experiment. After the hybridization with the specific BCL-2 cDNA probe (21) a $5.4 \mathrm{~kb}$ band corresponding to the BCL-2 mRNA was detected in all the samples. To normalize the possible differences due to the mRNA loading and transfer, the same filters were de-hybridized and re-hybridized again with the human 28S rRNA cDNA probe (22). The ratio between the target mRNA level and the 28S rRNA level was calculated for each sample. The semi-quantitative expression of BCL-2 mRNA level in neoplastic prostate tissues was calculated as percentage of normal prostate mRNA level measured on the same filter. The mean values of at least three separate measurements for each patient were recorded and the average values $( \pm$ SEM) are reported in Fig. 1B. 
Table I. Clinicopathological features of 43 prostate tissue samples.

\begin{tabular}{|c|c|c|c|c|}
\hline Patients & Age & Gleason's grade & Hormonal therapy (months) & Hormonal therapy \\
\hline N1 & 69 & Control & - & - \\
\hline N2 & 63 & Control & - & - \\
\hline N3 & 80 & Control & - & - \\
\hline $\mathrm{N} 4$ & 83 & Control & & - \\
\hline $\mathrm{K} 1$ & 61 & 6 & - & - \\
\hline $\mathrm{K} 2$ & 73 & 9 & - & - \\
\hline K3 & 70 & 8 & - & - \\
\hline K4 & 59 & 7 & - & - \\
\hline K5 & 69 & 9 & - & - \\
\hline K6 & 61 & 9 & - & - \\
\hline K7 & 71 & 6 & - & - \\
\hline K8 & 63 & 8 & - & - \\
\hline K9 & 58 & 7 & - & - \\
\hline K10 & 64 & 6 & - & - \\
\hline K11 & 59 & 8 & - & - \\
\hline K12 & 72 & 8 & - & - \\
\hline K13 & 71 & 8 & - & - \\
\hline K14 & 72 & 7 & - & - \\
\hline K15 & 70 & 8 & - & - \\
\hline K16 & 67 & 7 & - & - \\
\hline K17 & 61 & 9 & - & - \\
\hline K18 & 70 & 7 & - & - \\
\hline K19 & 70 & 7 & - & - \\
\hline $\mathrm{K} 20$ & 60 & 7 & - & - \\
\hline K21 & 67 & 5 & - & - \\
\hline TK1 & 70 & 6 & 1 & Goserelin + Bicalutamide \\
\hline TK2 & 55 & 8 & 1 & Goserelin + Bicalutamide \\
\hline TK3 & 63 & 8 & 1 & Goserelin + Bicalutamide \\
\hline TK4 & 71 & 7 & 1 & Goserelin + Bicalutamide \\
\hline TK5 & 73 & 6 & 1 & Goserelin + Bicalutamide \\
\hline TK6 & 68 & 6 & 1 & Goserelin + Bicalutamide \\
\hline TK7 & 69 & 7 & 1 & Goserelin + Bicalutamide \\
\hline TK8 & 77 & 10 & 1 & Goserelin + Bicalutamide \\
\hline TK9 & 73 & 6 & 1 & Goserelin + Bicalutamide \\
\hline TK10 & 70 & 7 & 1 & Goserelin + Bicalutamide \\
\hline TK11 & 70 & 9 & 1 & Goserelin + Bicalutamide \\
\hline TK12 & 63 & 7 & 3 & Goserelin + Bicalutamide \\
\hline TK13 & 63 & 8 & 3 & Goserelin + Bicalutamide \\
\hline TK14 & 76 & 3 & 3 & Goserelin + Bicalutamide \\
\hline TK15 & 60 & 9 & 6 & Goserelin + Bicalutamide \\
\hline TK16 & 68 & 6 & 6 & Goserelin + Bicalutamide \\
\hline TK17 & 75 & 7 & 6 & Goserelin + Bicalutamide \\
\hline TK18 & 68 & 8 & 6 & Goserelin + Bicalutamide \\
\hline
\end{tabular}

$\mathrm{N}$, normal prostate; $\mathrm{K}$, prostate cancer; TK, prostate cancer after neoadjuvant ADT.

The results of Northern hybridization experiments showed decreased BCL-2 steady-state mRNA level in $85 \%$ of the neo- plastic prostate tissues obtained from patients without therapy (K1-K13) when compared with normal prostate samples (N1-N4). 


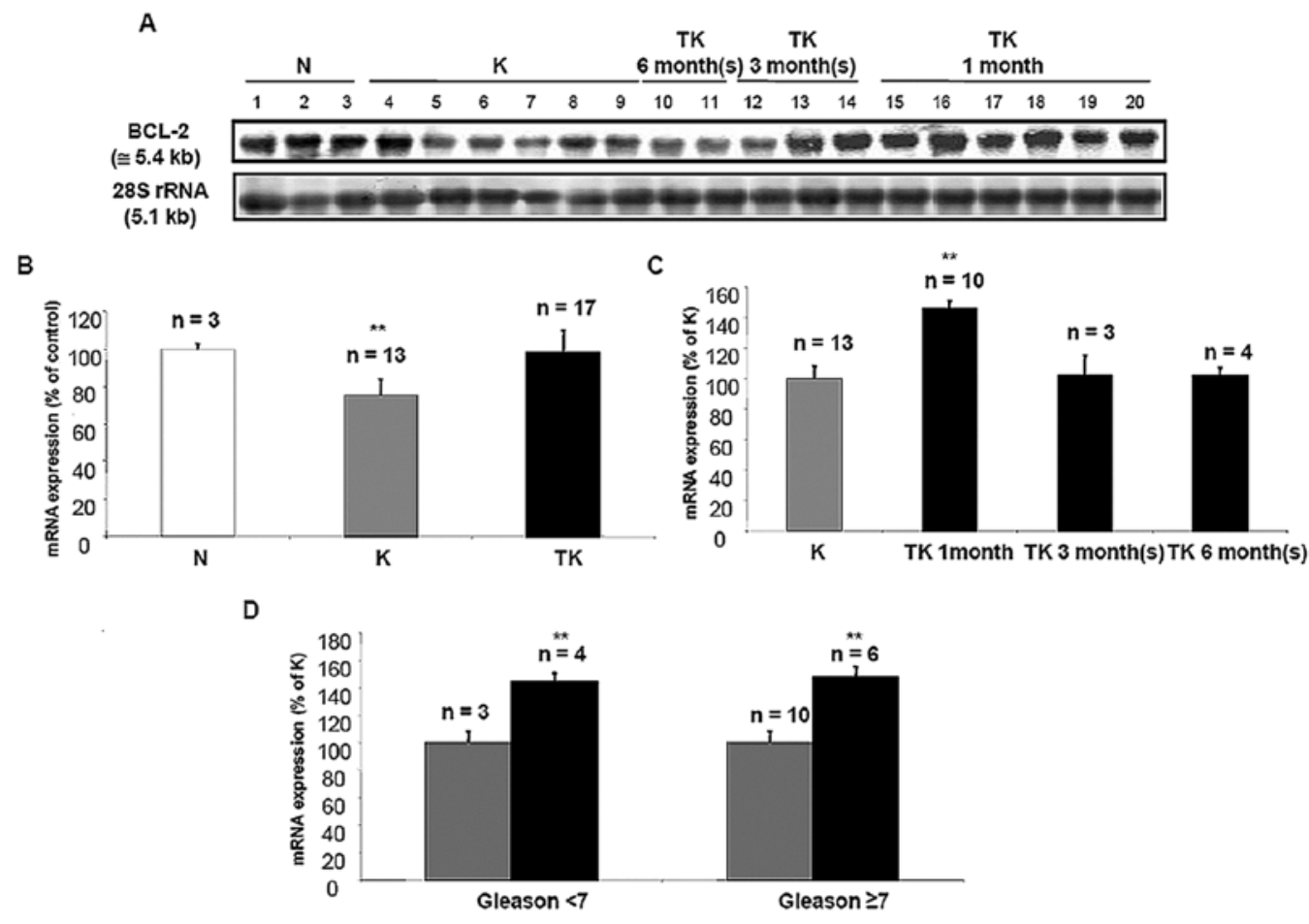

Figure 1. Effect of neoadjuvant ADT on the BCL-2 mRNA level. (A) Total RNA ( $25 \mu \mathrm{g})$ was isolated from prostate tissues. Lane 1-3, RNA isolated from 3 patients with normal prostate tissues. Lanes 4-9, RNA isolated from 6 patients with neoplastic prostate tissues. Lanes 10-11, RNA isolated from 2 patients with neoplastic prostate tissues who had undergone 6 months of hormonal therapy (TK 6 months). Lanes 12-14, RNA isolated from 3 patients with neoplastic prostate tissues who had undergone 3 months of hormonal therapy (TK 3 months). Lanes 15-20, RNA isolated from 6 patients with neoplastic prostate tissues who had undergone 1 month of hormonal therapy (TK 1 month). After hybridization with BCL-2 probe, the filter was stripped and hybridizated with $28 \mathrm{~S}$ rRNA probe. (B) The average of BCL-2 mRNA level in the samples of normal prostate tissues $(\mathrm{N})$ was set at 100, and the mRNA levels in the other samples $(\mathrm{K}$ and TK) were calculated as a percentage of N. (C) The average of BCL-2 mRNA level in neoplastic tissues isolated from patients treated with 1 month of hormonal therapy (TK 1 month), patients treated with 3 months of hormonal therapy (TK 3 months) and patients treated with 6 months (TK 6 months) of hormonal therapy were all calculated as a percentage of K. (D) The average of BCL-2 mRNA level during cancer progression in neoplastic tissues after 1 month of hormonal therapy (TK) were calculated for patients with Gleason $<7$ and patients with Gleason $>7\left(n=4\right.$ and $n=6$, respectively) as percentage of K. Error bars indicate SEM; ${ }^{*} \mathrm{P}<0.05 ;{ }^{* * *} \mathrm{P}<0.01 ; n=\mathrm{number}$ of patients analyzed.

On the contrary, an increased BCL-2 steady-state mRNA level in $59 \%$ of the neoplastic tissues isolated from patients who received neoadjuvant ADT (TK1-TK17) was observed.

The expression of BCL-2 mRNA in the prostate cancer samples isolated from patients without therapy $(\mathrm{K})$ ranged from $43 \%( \pm 13)$ to $95 \%( \pm 28)$, with an average value of $75 \%( \pm 9)$. This decrease was statistically significant $(\mathrm{p}<0.01)$ (Fig. 1B). On the contrary the BCL-2 mRNA level in the neoplastic tissues isolated from patients who received neoadjuvant ADT (TK) ranged between $51 \%( \pm 6)$ and $129 \%( \pm 21)$, with a mean percentage value of $98 \%( \pm 12)$.

Setting at 100 (arbitrary units) the average BCL-2 mRNA level in neoplastic prostate tissue specimens $(\mathrm{K})$, the BCL-2 mRNA level in neoplastic tissues isolated from patients who received neoadjuvant ADT (TK) was increased $(128 \pm 8 \%)$ with respect to the $\mathrm{K}$ group, and was statistically significant $(\mathrm{p}<0.01)$.

In conclusion, our data show that short-term administration of ADT interferes with BCL-2 mRNA expression, reverting the BCL-2 mRNA decrease observed in the malignant cell transformation, thus suggesting that androgen-mediated mechanisms may act through the BCL-2-mediated apoptotic pathways.

Effect of the time of neoadjuvant ADT administration. Since the neoadjuvant ADT is normally administered to patients affected by prostate cancer for different time periods we decided to investigate the effect of the length of administration time on the BCL-2 mRNA expression. In this regard, we evaluated BCL-2 mRNA level in the neoplastic prostate samples isolated from patients treated for 1, 3 and 6 months, respectively.

As shown in Fig. 1C, the BCL-2 mRNA expression measured in the neoplastic tissues isolated from patients treated with ADT for 1 month showed a statistically significant $(\mathrm{p}<0.01)$ increase $(146 \pm 12 \%)$ in comparison with neoplastic tissues isolated from patients without therapy $(\mathrm{K})$. On the contrary, BCL-2 mRNA expression in the patients treated with ADT for 3 and 6 months resulted comparable to the expression measured in the tumour tissues isolated from patients without therapy $(\mathrm{K})$.

Effect of ADT on clinical progression. To investigate whether the effect of 1 month of ADT on the BCL-2 mRNA expression associated with the aggressiveness of prostate cancer, we correlated the BCL-2 mRNA to the histological tumour grade based on Gleason's criteria of tumour classification (18).

As reported in Fig. 1D, the 4 TK patients affected by a well-differentiated prostate carcinoma (Gleason $<7$ ) and the 6 TK patients affected by poor-differentiated prostate carcinoma (Gleason $\geq 7$ ) showed a statistically significant $(\mathrm{p}<0.01)$ similar increase $(144 \pm 6 \%$ for Gleason $<7$ and $147 \pm 8 \%$ for Gleason $\geq 7$, 


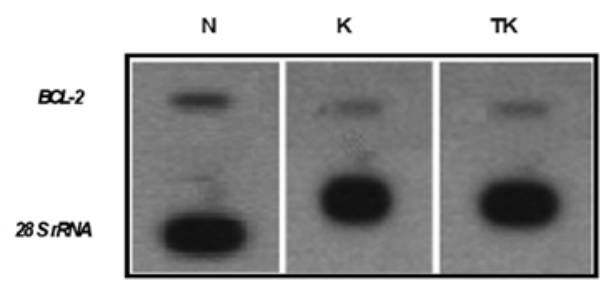

B

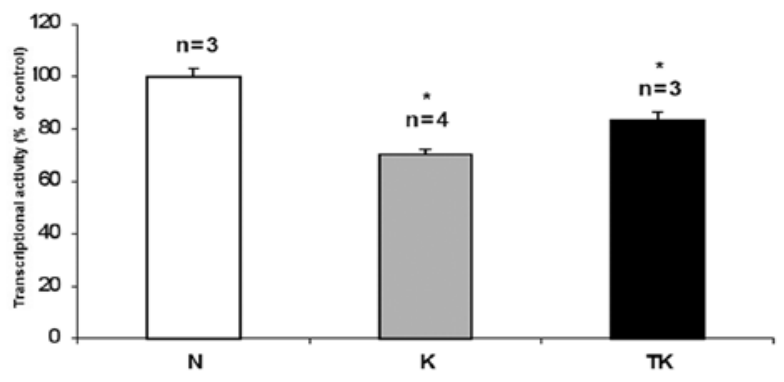

Figure 2. Transcriptional regulation of BCL-2 gene. The typical autoradiography of nuclear run on experiments for 1 representative normal prostate $(\mathrm{N}), 1$ representative neoplastic prostate $(\mathrm{K})$ and 1 representative neoplastic prostate after 1 month of hormonal therapy (TK) is shown (A). To normalize the amount of transcriptional activity for each sample, the filters were stripped and hybridized again using the $1.3 \mathrm{~kb}$ specific $28 \mathrm{~S}$ rRNA probe. After normalization BCL-2 (B) transcriptional activities in the 4 neoplastic human prostate specimens $(\mathrm{K})$ and in the 3 neoplastic tissues isolated from patients after 1 month of hormonal therapy (TK) were calculated as the percentile average of the 3 normal human prostate specimens $(\mathrm{N})$, transcriptional activities. Mean values \pm SEM from two different experiments are shown. Error bars indicate SEM. ${ }^{*} \mathrm{P}<0.05$.

respectively) of the BCL-2 mRNA expression in comparison to patients without therapy (K). Since comparing the BCL-2 mRNA expression in patients with Gleason $<7$ and patients with Gleason $\geq 7$ no statistically significant difference was found ( $p>0.05)$, even if these preliminary results need to be verified in a larger population, we can conclude that BCL-2 increase observed in the patients treated with neoadjuvant ADT for 1 month does not correlate with the tumour progression.

Effect of ADT on transcriptional regulation of BCL-2 gene. To investigate the molecular mechanism responsible for the variation of BCL-2 mRNA level in prostate cancer tissues isolated from patients treated with ADT for 1 month, we measured the transcriptional activity of the BCL-2 gene by nuclear Run-on assays.

A typical run-on experiment was carried out by using one representative normal prostate $(\mathrm{N})$, one representative neoplastic prostate sample isolated from patients without therapy $(\mathrm{K})$ and one representative neoplastic prostate specimen isolated from patients treated for 1 month with neoadjuvant ADT (TK), is shown in Fig. 2A.

In the neoplastic tissues isolated from patients who received neoadjuvant ADT for 1 month (TK) BCL-2 transcriptional activity ranged between $65 \%( \pm 2)$ and $84 \%( \pm 4)$, with a mean percentage value of $76 \%( \pm 3)$. In the prostate cancer samples isolated from 4 patients without therapy $(\mathrm{K})$ BCL-2 transcriptional activity ranged from $61 \%( \pm 1)$ to $80 \%$ $( \pm 6)$, with an average value of $72 \%( \pm 8)$.
A
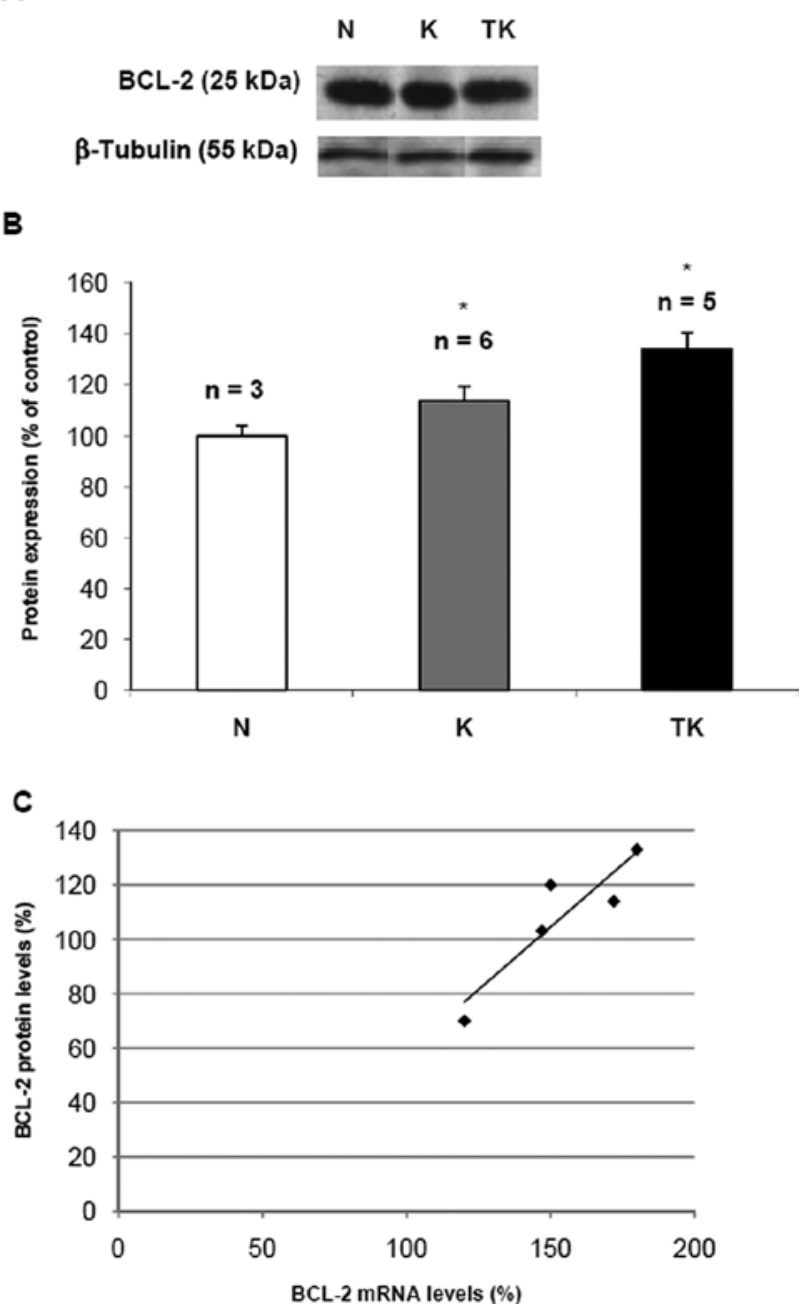

Figure 3. Effect of ADT on BCL-2 protein expression. A typical autoradiography of immunoblotting for 1 representative normal prostate $(\mathrm{N})$, for 1 representative neoplastic prostate $(\mathrm{K})$ and for 1 representative neoplastic prostate after 1 month of hormonal therapy (TK) is shown (A). To normalize the amount of total proteins loaded for each sample, the blots were stripped and re-hybridized using the $\beta$-tubulin antibody. (B) The average of BCL-2 protein expression level in 3 normal prostate tissue samples $(\mathrm{N})$ was set at 100, and BCL-2 protein level in the 6 neoplastic prostates $(\mathrm{K})$ and in the neoplastic prostates isolated from 5 patients after 1 month of hormonal therapy (TK) were calculated as percentage of N. (C) Regression analysis between protein level and mRNA level in prostate cancer tissues after 1 month of hormonal therapy showed positive correlation between BCL-2 protein level and BCL-2 mRNA expression levels: $\mathrm{r}=0.91 ; \mathrm{p}=0.016$. Mean values $\pm \mathrm{SEM}$, from at least two different experiments are shown; error bars indicate $\pm \mathrm{SEM}$; $\mathrm{P}<0.05$.

The decrease of the BCL-2 gene transcriptional activity was statistically significant $(\mathrm{p}<0.05)$ both in neoplastic prostate tissues isolated from patients without therapy $(\mathrm{K})$ and in neoplastic prostate tissues isolated from patients treated with neoadjuvant ADT for 1 month (TK) in comparison with the normal prostates (N) (Fig. 2B), suggesting that the BCL-2 transcriptional activity does not account for the up-regulation of the mRNA expression as measured by Northern hybridization experiments in neoplastic prostate tissues isolated from patients treated with neoadjuvant ADT for 1 month. On the contrary, our results suggests that transcriptional activity may be involved in the BCL-2 regulation of expression in neoplastic prostate tissues. 
A

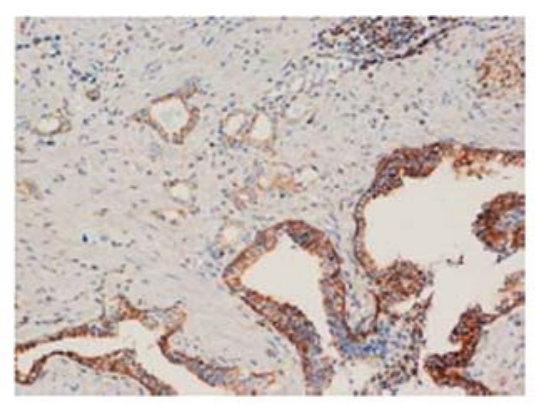

C

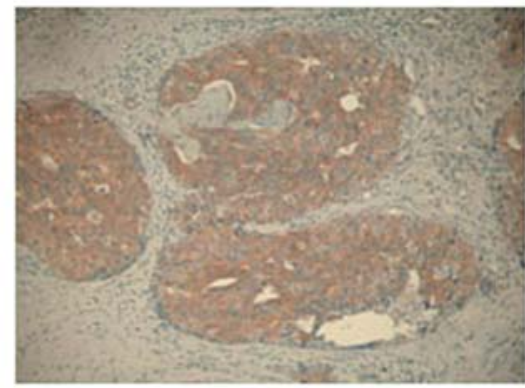

B

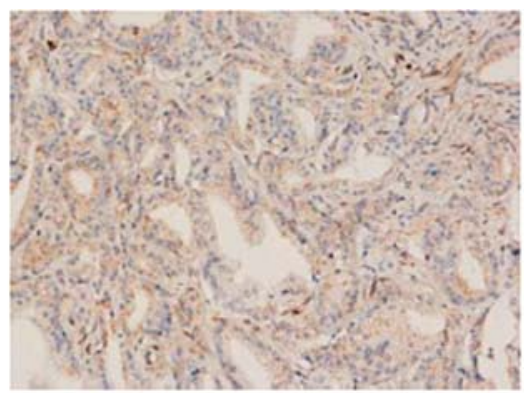

D

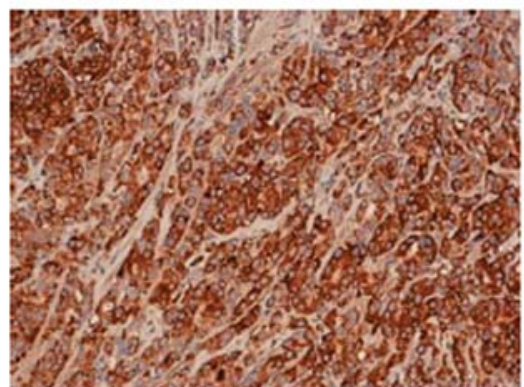

E

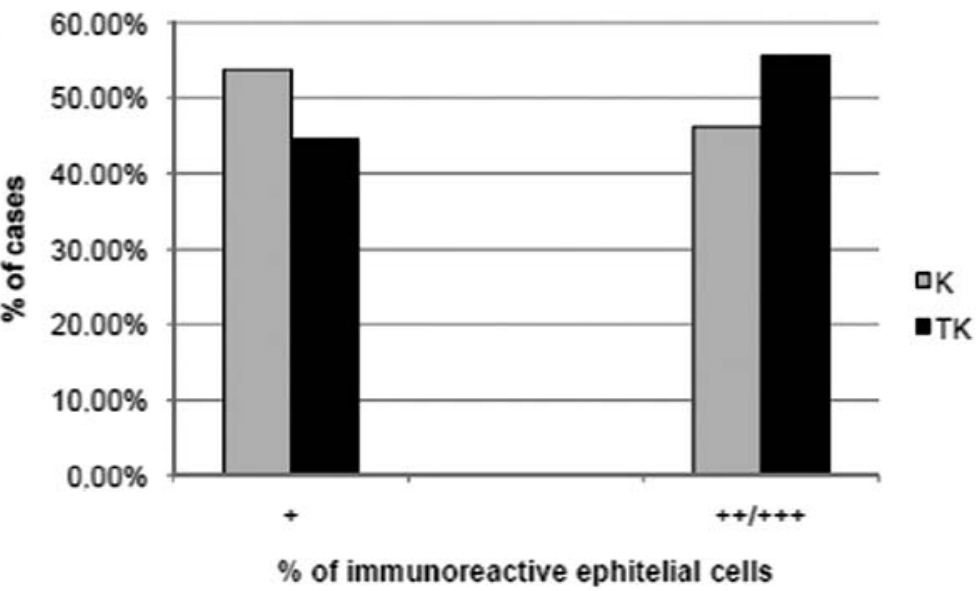

Figure 4. BCL-2 immunohistochemical analysis in neoplastic prostate tissues. ABC immunohistochemical technique of one representative neoplastic prostate tissue showing the BCL-2 distribution respectively in stromal (A, x20) and epithelial cells (B, x40). ABC immunohistochemical technique of one representative neoplastic prostate after 1 month of hormonal therapy showing the BCL-2 distribution respectively in stromal (C, x20) and epithelial cells (D, x40). The results of semi-quantitative evaluation of BCL-2 immunoreactive epithelial cells (E) calculated as: + , weak positivity of cells to BCL-2; ++/+++, temperate or acute positivity of cells to BCL-2, are reported for the prostate tissues from 14 patients affected by prostate cancer (K) and 9 patients affected by prostate cancer who underwent hormonal therapy (TK) for 1 month hormonal therapy (TK).

Effect of ADT on BCL-2 protein level. To clarify the molecular mechanisms that regulate the expression of BCL-2, we measured the BCL-2 protein level by Western blot analysis, in the prostate tissues isolated from patients treated with ADT for 1 month.

Fig. 3A shows the results of a typical experiment. After normalization with $\beta$-tubulin protein the mean value of the BCL-2 protein expression was calculated as percentage of the normal prostate. At least three separate measurements for each patient were recorded and the average \pm SEM are reported in Fig. 3B.

The BCL-2 protein expression in the prostate cancer samples isolated from patients without any therapy $(\mathrm{K})$ ranged from $95 \%( \pm 8)$ to $139 \%( \pm 3)$, with an average value of $114 \%( \pm 6)$ in comparison to the normal prostate control $(\mathrm{N})$. This up-regulation of BCL-2 protein level was statistically significant $(\mathrm{p}<0.05)$.
The BCL-2 protein level in the neoplastic tissues isolated from patients who received neoadjuvant ADT for 1 month (TK) ranged between $80 \%( \pm 4)$ and $220( \pm 10)$, in comparison to the normal prostate control $(\mathrm{N})$, with a mean percentage value of $134 \%( \pm 7)$ and this up-regulation resulted to be statistically significant $(\mathrm{p}<0.05)$.

Moreover, the BCL-2 protein level in neoplastic tissues isolated from patients treated with neoadjuvant ADT for 1 month (TK) was increased $(120 \pm 10 \%)$ also with respect to the $\mathrm{K}$ group and this increase was statistically significant $(\mathrm{p}<0.05)$.

Our data, together with the results obtained from the run-on experiments suggest that translational and/or post-translational regulation take place in BCL-2 expression in neoplastic prostate tissues depending from ADT administration for 1 month. The increase in BCL-2 proteins level directly correlated with the up-regulation of BCL-2 mRNA steady-state expression in TK group $(r=0.91 ; p=0.016)$ as shown in Fig. 3C. 
We also investigated the BCL-2 expression by immunohistochemistry in the neoplastic tissues. The study material included 22 surgical prostate samples of 13 patients with carcinoma $(\mathrm{K})$ and 9 patients treated for 1 month with ADT (TK).

In both $\mathrm{K}$ and TK groups, BCL-2 localization was detected mainly in the epithelial compartment (compare Fig. 4A and C and the 40x magnification $B$ and $D$, respectively).

The BCL-2 immunohistochemical staining pattern (BCL-2 positive) in K group was as follows: in 7 of 13 patients $(53.80 \%)$ the positivity of BCL-2 immunoreactivity resulted weak and in 6 of 13 patients (46.20\%) the positivity of BCL-2 immunoreactivity was temperate or acute (Fig. 4B).

On the contrary, the BCL-2 immunohistochemical staining pattern (BCL-2 positive) in TK group was as follows: in 4 of 9 patients (44.40\%) the positivity of BCL-2 immunoreactivity resulted weak and in 5 of 9 patients $(55.60 \%)$ the positivity of BCL-2 immunoreactivity was temperate or acute (Fig. 4D).

In conclusion, in the greater percentage of TK patients (55.60\%) showing a temperate/acute BCL-2 positivity, we observed an increase of BCL-2 expression depending from the ADT administration for 1 month (Fig. 4E).

\section{Discussion}

In prostate cancer, BCL-2 has been reported to suppress apoptosis induction after androgen ablation both in vitro and in vivo $(11,23,24)$. BCL-2 is not strongly expressed in normal human prostate tissue but it is highly expressed in androgen-independent tumors $(25,26)$. BCL-2 expression in human androgen sensitive prostate cancers, correlates with the subsequent development of androgen-independence and has been candidate as a prognostic indicator for the development of androgen escape in organ-confined prostate tumors $(24,27,28)$. Transfection with BCL-2 oncogene, resulting in BCL-2 protein overexpression, has showed to induce resistance to the hormone therapies in androgen-sensitive cell lines and was found to protect these cells against apoptosis (29). Moreover, animal studies showed that BCL-2 expression increases with the development of hormonerefractory disease after surgical castration (11). Mechanism by which BCL-2 expression is up-regulated during the development of hormone-refractory disease, are unknown, although it has been proposed that androgens are capable of suppressing BCL-2 expression; therefore after androgen withdrawal BCL-2 expression increases. This is supported by the fact that in the $\mathrm{LNCaP}$ cell line, dihydrotestosterone suppresses BCL-2 expression (11).

On the other hand BCL-2 activity in androgen-independent prostate cancer does not necessarily have to occur through overexpression. For example, activation of PI3K/Akt pathway results in activated BCL-2. Activation of PI3K/Akt pathway, through the loss of PTEN, results in the BAD phosphorylation. This frees BCL-2 from BAD-BCL-2 complex, which ultimately leads to cell survival (30).

In the present study we investigated the cross-talk between hormones and anti-apoptotic factors by studying the effect of ADT treatment on BCL-2 expression in patients affected by prostate cancer.

We show for the first time, a significant decrease of BCL-2 mRNA levels in prostate cancer (group K) compared with the normal prostate tissues (group N). On the contrary, considering the BCL-2 mRNA levels in patients who received neoadjuvant
ADT (group TK) we observed a statistically significant increase in comparison to patients who did not undergo hormonal treatment (group K), which shows the BCL-2 mRNA levels comparable to those of normal tissue.

Our results suggest that the prostate tumor cells in some way inhibits the synthesis of BCL-2 mRNA, but this effect disappears in the presence of chemotherapeutic factors that inhibit the action of the androgens on the prostate cell. Therefore, we can hypothesize that in prostate cancer ADT may possible reverting the inhibitory effect on BCL-2 expression.

Since the neoadjuvant ADT is normally administered to patients affected by prostate cancer for different time periods, we evaluated BCL-2 mRNA levels in the neoplastic prostate samples isolated from patients treated for 1, 3 and 6 months, respectively. In the present study we demonstrated that the BCL-2 mRNA expression measured in the neoplastic tissues isolated from patients treated with ADT for 1 month showed a statistically significant increase in comparison with neoplastic tissues isolated from patients without therapy $(\mathrm{K})$. On the contrary, BCL-2 mRNA expression in the patients treated with ADT for 3 and 6 months resulted comparable to the expression measured in the tumour tissues isolated from patients without therapy $(\mathrm{K})$.

These results suggest that ADT interferes with BCL-2 expression in a positive regressive way only with administration for short time (1 month), possibly indicating that androgenmediated mechanisms act through pathways involving BCL-2 in the first phase of the disease.

It was previously shown that increased levels of BCL-2 correlates with the development of prostate cancers, and suggested that this survival factor associates with a hormone-insensitive, metastatic phenotype (6). In this light, we evaluated the association between the effect of 1 month ADT on the BCL-2 mRNA levels and the tumor aggressiveness. Comparing the BCL-2 mRNA expression in TK patients with Gleason $<7$ and patients TK with Gleason $\geq 7$ no statistically significant difference was found ( $\mathrm{p}>0.05)$.

Even if these preliminary results need to be verified in a larger population, we can conclude that the BCL-2 increase observed in the patients treated with neoadjuvant ADT for 1 month does not correlated with the tumour progression.

To investigate the molecular mechanisms responsible of the BCL-2 gene regulation of expression, we carried out in vivo transcriptional assays by means of run-on experiments. Our results suggest that the BCL-2 transcriptional activity does not account for the up-regulation of the mRNA expression as measured by Northern hybridization experiments in neoplastic prostate tissues isolated from patients treated with neoadjuvant ADT for 1 month. On the contrary, our data suggest that an inhibition of the transcriptional activity may be observed for the BCL-2 down-regulation of expression observed in neoplastic prostate tissues.

The post-transcriptional mechanisms are involved in the regulation of BCL-2 mRNA expression by ADT, thus we also measured the BCL-2 protein content by means of Western blot analysis.

Our results showed an increase of the BCL-2 protein expression in the prostate cancer samples isolated from patients without therapy $(\mathrm{K})$ in agreement with previous data showing an increase of BCL-2 protein level in an in vitro system $(7,8)$. 
The BCL-2 protein level resulted up-regulated also in the neoplastic tissues isolated from patients who received neoadjuvant ADT for 1 month (TK) in comparison to both the neoplastic prostate tissues obtained from patients without therapy $(\mathrm{K})$ and the normal prostate samples $(\mathrm{N})$. These data, together with the results obtained from the run-on experiments suggest a translational and/or post-translational regulation of BCL-2 expression induced by ADT treatment.

Moreover, the results of immunohistochemistry showed a greater percentage of BCL-2 expression in the epithelial compartment of prostate section isolated from patients who received neoadjuvant ADT for 1 month (TK) in comparison to the patients without therapy. These data are in agreement with other laboratory results showing a correlation between the presence of BCL-2 protein and the anti-androgen therapy (4), suggesting that BCL-2 probably acts in an autocrine way on the prostate cancer cells deprived of androgens.

In conclusion, our data show that short-term administration of ADT interferes with BCL-2 expression, suggesting that androgen-mediated mechanisms may act through the BCL-2 mediated apoptotic pathways.

Our data seem to indicate that ADT short-term administration does not interfere with BCL-2 expression at transcriptional level, suggesting that androgen-mediated mechanisms involving BCL-2 pathways, probably act at posttranscriptional level.

Precise characterization of the mechanisms that regulate the expression of BCL-2 in prostate cancer cells will provide further insight into the molecular basis involved in malignant transformation and disease progression and possibly contribute to the identification of molecular targets for the development of new therapeutic strategies for cancer disease.

Finally, a better understanding of the cross-talk among apoptotic factors such as BCL-2 and hormones during the clinical progression of prostate cancer is critical not only to contribute to the understanding of the molecular mechanisms that support malignant cell transformation and progression, metastasis establishment and growth, but also to identify new targeted modulators of cell proliferation that may help in generating rationally designed drugs or a gene therapy based on tissue specific expression of these factors in order to control cancer cell proliferation. Recent studies confirmed that inhibition of BCL-2 function, by combined antisense BCL-2 ODN and chemotherapeutic agent after castration, delays progression to androgen-independent and inhibition of established androgen- independent tumor growth in mice with Shionigi tumor $(16,17)$.

Whether it is antisense, gene therapy or drug therapy, an effective anti-BCL-2 treatment of androgen-independent tumors may also potentiate the apoptosis inducing ability of other agents $(16,17)$ or radiotherapy $(18)$ that otherwise cannot overcome the survival signal trigger by BCL-2 overexpression.

\section{References}

1. Akao Y, Otsuki Y, Kataoka S, Ito Y and Tsujimoto Y: Multiple subcellular localization of BCL-2: detection in nuclear outer membrane, endoplasmic reticulum membrane, and mitochondrial membranes. Cancer Res 54: 2468-2471, 1994.

2. Fulda S, Gorman AM, Hori O and Samali A: Cellular stress responses: cell survival and cell death. Int J Cell Biol 2010: 1-23, 2010 .
3. Danial NN: BCL-2 family proteins: critical checkpoints of apoptotic cell death. Clin Cancer Res 13: 7254-7263, 2007.

4. McDonnell TJ, Troncoso P, Brisbay SM, Logothetis C, Chung LW, Hsieh JT, et al: Expression of the protooncogene BCL-2 in the prostate and its association with emergence of androgen-independent prostate cancer. Cancer Res 52: 6940-6944, 1992

5. Colombel M, Symmans F, Gil S, O'Toole KM, Chopin D, Benson M, et al: Detection of the apoptosis-suppressing oncoprotein BCL-2 in hormone-refractory human prostate cancers. Am J Pathol 143: 390-400, 1993.

6. Krajewska M, Krajewski S, Epstein JI, Shabaik A, Sauvageot J, Song K, et al: Immunohistochemical analysis of BCL-2, bax, BCL-x and mcl-1 expression in prostate cancers. Am J Pathol 148: 1567-1576, 1996.

7. McConkey DJ, Greene G and Pettaway CA: Apoptosis resistance increase with metastatic potential in cells of the human LNCaP prostate carcinoma line. Cancer Res 56: 5594-5599, 1996.

8. Lu S, Tsai SY and Tsai MJ: Molecular mechanisms of androgenindependent growth of human prostate cancer LNCaP-AI cells. Endocrinology 140: 5054-5059, 1999.

9. Feldman BJ and Feldman D: The development of androgen independent prostate cancer. Nat Rev Cancer 1: 34-45, 2001.

10. Raffo AJ, Perlman H, Chen MW, Day ML, Streitman JS and Buttyan R: Overexpression of BCL-2 protects prostate cancer cells from apoptosis in vitro and confers resistance to androgen depletion in vivo. Cancer Res 55: 4438-4445, 1995.

11. Bruckheimer EM, Spurgers K, Weigel NL, Logothetis C and McDonnell TJ: Regulation of BCL-2 expression by dihydrotestosterone (DHT) in hormone sensitive LNCaP-FGC prostate cancer cells. J Urol 169: 1553-1557, 2003.

12. Yamanaka K, Rocchi P, Miyake H, Fazli L, Vessella B, Zangemeister-Wittke U, et al: A novel antisense oligonucleotide inhibiting several antiapoptotic BCL-2 family members induces apoptosis and enhances chemosensitivity in androgen-independent human prostate cancer PC3 cells. Mol Cancer Ther 4: 1689-1698, 2005

13. Yamanaka K, Rocchi P, Miyake H, Fazli L, So A, ZangemeisterWittke U, et al: Induction of apoptosis and enhancement of chemosensitivity in human prostate cancer $\mathrm{LNCaP}$ cells using bispecific antisense oligonucleotide targeting BCL-2 and BCL-xL genes. BJU International 97: 1300-1308, 2006.

14. Haldar S, Jena N and Croce CM: Inactivation of BCL-2 by phosphorylation. Proc Natl Acad Sci USA 92: 4507-4511, 1995.

15. Yoshino T, Shiina H, Urakami S, Kikuno N, Yoneda T, Shigeno K, et al: BCL-2 expression as a predictive marker of hormone-refractory prostate cancer treated with taxane-based chemotherapy. Clin Cancer Res 12: 6116-6124, 2006.

16. Gleave ME, Miayake H, Goldie J, Nelson C and Tolcher A: Targeting BCL-2 gene to delay androgen-independent progression and enhance chemosensitivity in prostate cancer using antisense BCL-2 oligodeoxynucleotides. Urology 54: 36-46, 1999.

17. Miayake $\mathrm{H}$, Tolcher A and Gleave ME: Chemosensitization and delayed androgen-independent recurrence of prostate cancer with the use of antisense BCL-2 oligodeoxynucleotides. J Natl Cancer Inst 92: 34-41, 2000.

18. Pollack A, Cowen D, Troncoso P,Zagars GK, von Eschenbach AC, Meistrich ML, et al: Molecular markers of outcome after radiotherapy in patients with prostate carcinoma. Cancer 97: 1630-1638, 2003.

19. Gleason DF: Histologic grading of prostate cancer: a perspective. Hum Pathol 23: 273-279, 1992.

20. Perlino E, Lovecchio M, Vacca RA, Fornaro M, Moro L, Ditonno P, et al: Regulation of mRNA and protein levels of betal integrin variants in human prostate carcinoma. Am J Pathol 157: 1727-1734, 2000.

21. Fuzio P, Lucarelli G, Perlino E, Battaglia M, Bettocchi C, Selvaggi FP, et al: Androgen deprivation therapy regulation of beta1C integrin expression in prostate cancer. Oncol Rep 22: 327-335, 2009.

22. Bhatia P, Taylor WR, Greenberg AH and Wright JA: Comparison of glyceraldehyde-3-phosphate dehydrogenase and 28S-ribosomal RNA gene expression as RNA loading controls for Northern blot analysis of cell lines of varying malignant potential. Anal Biochem 216: 223-226, 1994

23. Rothermund CA, Kondrikov D, Lin MF and Vishwanatha JK: Regulation of Bcl-2 during androgen-unresponsive progression of prostate cancer. Prostate Cancer Prostatic Dis 5: 236-245, 2002.

24. Szostak MJ, Kaur P, Amin P, Jacobs SC and Kyprianou N: Apoptosis and bcl-2 expression in prostate cancer: significance in clinical outcome after brachytherapy. J Urol 165: 2126-2130, 2001. 
25. Ohigashi T, Ueno M, Nonaka S, Nakanoma T, Furukawa Y, Deguchi N, et al: Tyrosine kinase inhibitors reduce bcl-2 expression and induce apoptosis in androgen-dependent cells. Am J Physiol Cell Physiol 278: C66-C72, 2000.

26. Rosser CJ, Reyes AO, Vakar-Lopez F, Levy LB, Kuban DA Hoover DC, et al: Bcl-2 is significantly overexpressed in localized radio-recurrent prostate carcinoma, compared with localized radio-naive prostate carcinoma. Int J Radiat Oncol Biol Phys 56: $1-6,2003$.

27. Wu TT, Hsu YS, Wang JS, Lee YH and Huang JK: The role of p53, bcl-2 and E-cadherin expression in predicting biochemical relapse for organ confined prostate cancer in Taiwan. J Urol 170 : $78-81,2003$
28. Herzig MC, Trevino AV, Liang H, Salinas R, Waters SJ, MacDonald JR, et al: Apoptosis induction by the dual-action DNA- and protein-reactive antitumor drug irofulven is largely Bcl-2-independent. Biochem Pharmacol 65: 503-513, 2003.

29. Huang JM, Lin TY, Chang D, Lin SL and Ying SY: Truncated Bcl-2, a potential pre-metastatic marker in prostate cancer. Biochem Biophys Res Commun 306: 912-917, 2003.

30. Datta SR, Dudek H, Tao X, Masters S, Fu H, Gotoh Y, et al: Akt phosphorylation of BAD couples survival signals to the cellintrinsic death machinery. Cell 91: 231-241, 1997. 